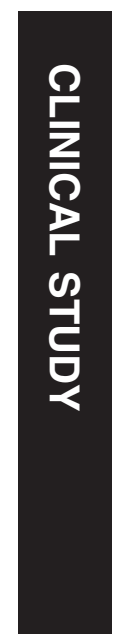

${ }^{1}$ Department of Ophthalmology, Soonchunhyang University Bucheon Hospital, Soonchunhyang University College of Medicine, Bucheon, Korea

${ }^{2}$ Department of Endocrinology, Brain Korea 21 Project for Medical Science, Institute of Endocrine Research, and Severance Integrative Research Institute for Cerebral and Cardiovascular Disease, Yonsei University College of Medicine, Seoul, Korea

${ }^{3}$ The Institute of Vision Research, Department of Ophthalmology, Yonsei University College of Medicine, Seoul, Korea

Correspondence: JS Yoon, The Institute of Vision Research, Department of Ophthalmology, Yonsei University College of Medicine, 50 Yonsei-Ro, Seodaemun-ku, 120-752 Seoul, Korea.

Tel: +82 22228 3570;

Fax: +82 2312 0541;

E-mail: yoonjs@yuhs.ac

Received: 9 January 2013 Accepted in revised form: 30 March 2013 Published online: 7 June 2013

\section{Relevance of TSH-receptor antibody levels in predicting disease course in Graves' orbitopathy: comparison of the third-generation TBII assay and Mc4-TSI bioassay}

\begin{abstract}
Aims To investigate if TSH-receptor antibody (TRAb) levels measured in early Graves' orbitopathy (GO) stages are predictive of clinical disease course beyond 1 year after initial GO diagnosis and to compare performance of two newly developed TRAb assays (third-generation thyrotropin-binding inhibitor immunoglobulin (TBII) assay vs Mc4thyroid-stimulating immunoglobulin (TSI) bioassay) in predicting disease course. Methods Newly diagnosed, untreated GO patients whose duration of ocular symptoms was less than 6 months were included. One year after initial diagnosis, all patients were classified as presenting either a mild (Group 1) or severe course (Group 2) according to their clinical manifestations. The measurements of two TRAb assays at initial GO diagnosis were used for analysis. Results Data from 112 patients were available for analysis. Seventy-three patients (65.2\%) were designated as Group 1, and 39 patients $(34.8 \%)$ as Group 2. Patients with higher initial TRAb levels demonstrated a higher risk of severe disease course upon multiple regression analysis $(P<0.01)$. The cutoff values for the prediction of severe course of the third-generation TBII and Mc4-TSI assays were $10.67 \mathrm{IU} / 1$ and $555.10 \%$, respectively, with assay specificities of $\mathbf{8 4 . 9}$ and $89.0 \%$. The TBII assay predictive power
\end{abstract}

SY Jang ${ }^{1}$, DY Shin², EJ Lee 2 , SY Lee ${ }^{3}$ and JS Yoon ${ }^{3}$

(area under the curve $(\mathrm{AUC})=\mathbf{0 . 8 1 7} ; \mathbf{9 5 \%}$ confidence interval $(\mathrm{CI})=0.732-0.902)$ was equivalent to the TSI bioassay (AUC $=0.868$, 95\% $\mathrm{CI}=0.803-0.934)(P=0.203)$. Conclusions The predictive power of the third-generation TBII assay and Mc4-TSI bioassay are similarly strong. Measurement of TRAb using either third-generation TBII or Mc4-TSI in early GO periods would provide important prognostic information on future GO course.

Eye (2013) 27, 964-971; doi:10.1038/eye.2013.120; published online 7 June 2013

Keywords: Graves' orbitopathy; TSH-receptor antibody; clinical course; prediction

Introduction

The clinical course of Graves' orbitopathy (GO) is heterogeneous. The natural course is benign in a considerable proportion of GO cases. However, some GO cases may present with significant severe sequelae. The GO ocular manifestations include eyelid retraction, proptosis, chemosis, periorbital edema, altered ocular motility, and loss of vision with significant functional, social, and cosmetic consequences. ${ }^{1-4}$ As there are few clues currently available to assist clinicians in estimating GO prognosis, it is very difficult to predict which patients will suffer from such 
severe sequelae. Recently, Eckstein et al ${ }^{5}$ reported that persistently high TSH-receptor antibody (TRAb) levels were associated with a severe course of GO by demonstrating that TRAb levels at 12-24 months after diagnosis were significantly higher in patients with a severe course of GO than in those with a mild outcome. In their report, TRAb was measured using the second-generation thyrotropin-binding inhibitor immunoglobulin (TBII) assay.

Currently, there are two assays that are able to measure TRAb: one is the TBII assay and the other is a functional thyroid-stimulating immunoglobulin (TSI) bioassay. The TBII assay utilizes the ability of TRAb to inhibit the binding of radiolabeled TSH to TSH receptors. Through first-, second-, and third-generation modification, TBII has been modified to enhance its diagnostic sensitivity and specificity for Graves' disease (GD) ${ }^{6-8}$ Classical TBII assays are limited by their inability to discriminate between thyroid-stimulating antibodies (TSAbs) and thyroid-blocking antibodies (TBAbs). However, the most newly developed thirdgeneration TBII assay inhibits binding of a labeled TSAb (monoclonal Ab clone \#M22) rather than labeled TSH to the TSH receptor ${ }^{9,10}$ This results in enhanced sensitivity and specificity $v$ s earlier assays using radiolabeled TSH. 6,8,11,12 The TSI bioassay measures cyclic adenosine monophosphate production after TSAb binds to TSH receptor. ${ }^{13-15}$ Thus, this method enables the identification of the functional characteristics of TRAb. With the development of the Mc4-CHO cell line, the cell culture protocols of the TRAb bioassays were simplified. ${ }^{15-17}$ The superiority of the Mc4-CHO TSI bioassay has been reported with respect to GD diagnosis, ${ }^{17}$ and in the differential diagnosis of GD vs painless thyroiditis. ${ }^{16}$

Recent reports evaluated the relationship between Mc4-CHO TSI and GO. Lytton et al ${ }^{17}$ reported the superiority of the Mc4-CHO TSI bioassay in reflecting GO clinical activity and severity at a given time point, vs the second-generation TBII assay. Ponto et al ${ }^{18}$ also showed that the Mc4-CHO TSI assay results were better associated with GO clinical features than those of the second-generation TBII assay in their cross-sectional study. The correlation between TRAb levels and GO symptomology at certain time points may be of scientific importance, but may be limited in improving patient clinical management.

We investigated whether serum TRAb in newly diagnosed, untreated GO patients are predictive of disease course beyond 1 year after initial GO diagnosis. In addition, we compared the prognostic performance levels of two newly developed TRAb assays (a thirdgeneration TBII assay and a Mc4-CHO TSI bioassay) in predicting GO disease course.

\section{Patients and methods}

\section{Subjects}

This was a retrospective, consecutive, and observational study. Subjects were consecutive Korean GO patients attending the Department of Ophthalmology at the Severance Hospital of Yonsei University, College of Medicine, between January 2008 and May 2011. All subjects were newly diagnosed with GO, and no one had been previously treated with steroids or radiation before first presentation. All subjects met the following criteria: (1) the duration of GO ocular symptoms at initial diagnosis was no longer than 6 months; (2) both the third-generation TBII and Mc4-CHO TSI assays were performed simultaneously at initial GO diagnosis; and (3) patients were evaluated at regular intervals for longer than 1 year by a single ophthalmologist. Data from 112 patients were available for analysis. Past medical history, smoking history, GO duration, GD duration, type of current GD treatment, family history, presence of increased thyroid size, and peripheral thyroid function (initial free T4 level) were assessed. During this study, all patients with a clinical activity score (CAS; detailed below) of 4 or greater were prescribed steroid treatment. Steroid therapy usually began with oral prednisolone ( $1 \mathrm{mg} / \mathrm{kg}$ of body weight for adults and $0.5 \mathrm{mg} / \mathrm{kg}$ for children), which was tapered according to GO clinical activity and severity. Oral steroid administration was the preferred dosing regimen in our institute, because it was not necessary for patients to visit the clinic once a week. ${ }^{19}$ For severe cases, such as vision-threatening compressive optic neuropathy, intravenous steroids were given (methylprednisolone $500 \mathrm{mg}$ /day for 3 days), and then substituted with the tapered oral steroid regimen.

The study adhered to the Declaration of Helsinki, and approval to conduct this study was obtained from the Institutional Review Board of Severance Hospital of Yonsei University. Informed consent was obtained from all participants.

\section{Ocular examinations}

At each visit, the patients were examined for bestcorrected visual acuity, intraocular pressure measurement, and an exophthalmometry measured with a Hertel exophthalmometer. In patients with diplopia, Hess screen test, binocular single vision test, and computed tomography scan were performed. Monocular excursion was evaluated with the Hess screen test. GO activity was assessed using 7 points of the modified CAS as described by Mourits et al. ${ }^{20} \mathrm{GO}$ severity was assessed using the modified NOSPECS classification suggested by Eckstein et al.5,21 The sum of scores in each class constitutes the total NOSPECS score. Patient CAS and 
NOSPECS scores were evaluated by a single examiner who was blinded to the TRAb data (SYJ).

\section{Grouping subjects}

All patients were assigned to either the mild or severe GO category according to their ocular manifestations after 1 year beyond the initial visit. Patient grouping was done as described by Eckstein et al. 5,21 Patients with a CAS score of less than 4 and a NOSPECS score of less than 5 were assigned into the mild course group (Group 1 ), while patients with a CAS score equal or more than 4 and/or a NOSPECS score of 5 were defined as the severe course group (Group 2). Group 1 patients were in an inactive phase and displayed mild symptoms of GO; while Group 2 patients were considered to be in an active phase (GO activity was poorly controlled) and/or displayed severe GO sequelae. Patient CAS and NOSPECS scores were evaluated by a single examiner (SYJ) who was blinded to the TRAb data. One clinician who was blinded to the results of TRAb levels also performed a patient grouping.

\section{TSH-R antibody detection assays}

We used the measurement at initial GO diagnosis (initial TRAb levels) to analyze. Schematics depicting the TRAb assays are shown in Figure 1. TRAb was measured with a third-generation TBII assay using the automated Cobas electrochemiluminescence immunoassay (Elecsys, Roche Diagnostics GmbH, Penzberg, Germany) according to the manufacturer's instructions. Mc4-CHO TSI in patient sera was measured with the Thyretain TSI Reporter
BioAssay (Diagnostic Hybrids Inc., Athens, OH, USA) according to the manufacturer's instructions.

\section{Data analysis and statistics}

Normality of data distribution for all variables was tested using the Kolmogorov-Smirnov test. We used independent $t$-tests and Pearson $\chi^{2}$ analysis to compare primary data such as demographics and laboratory data between the two subject groups. Wilcoxon rank-sum test was used to compare median values of initial CAS score, duration of GO, duration of GD, duration of anti-thyroid drug (ATD) treatment, and follow-up periods between the two groups.

We investigated the relationships between initial TRAb levels and GO outcome using multiple logistic regression analysis, allowing for adjustment of possible factors influencing GO course. For multiple logistic regression analysis, TBII and TSI assays were entered into the model I and II, respectively. Models I and II were used to allow for adjustment of possible risk factors influencing GO course such as smoking, type of GD treatment, thyroid size, and steroid use. If there was a significant correlation $(R>0.5)$ between risk factors, only one factor was entered into the model to avoid multicollinearity. Receiver operating characteristic (ROC) plot analysis was performed to obtain the area under the ROC curve (AUC) using the third-generation TBII and Mc4-TSI assay values as independent variables, and the severe course of GO as dependent variables. Comparison of AUCs was performed using the Delong method. The sensitivity and specificity of both assays were examined at an optimal cutoff point in the ROC curves regarding the ability of the two assays in terms of discrimination
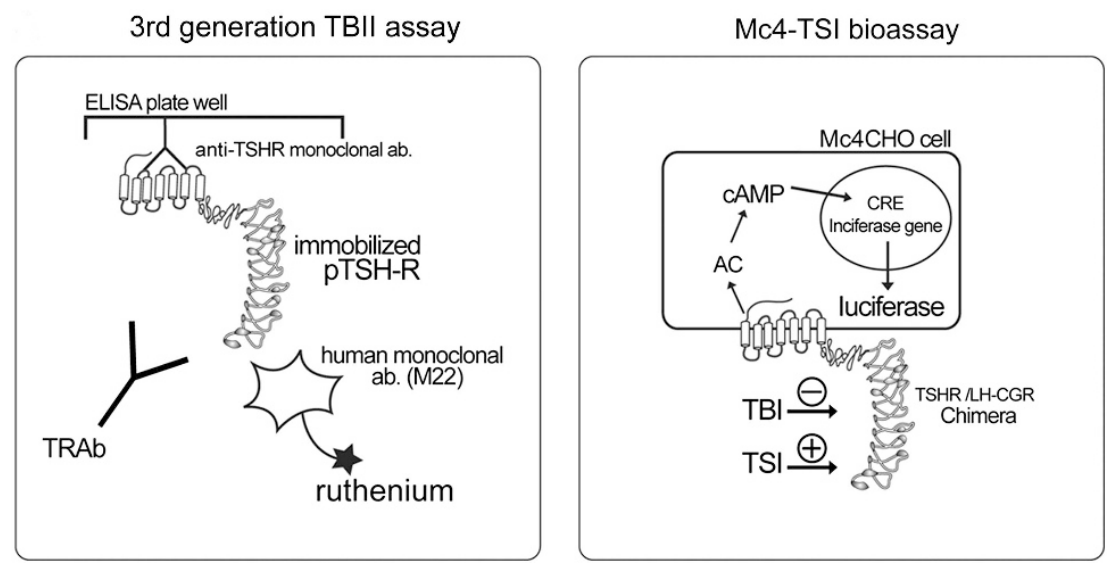

Figure 1 Schematic representation showing the principles of the third-generation TBII assay and Mc4-TSI bioassay. AC, adenylate cyclase; CRE, cAMP response element; LH-CG, lutropin-choriogonadotropin; TSH, thyroid-stimulating hormone; TRAb, TSH-receptor autoantibody; pTSH-R, porcine thyroid-stimulating hormone receptor; TSI, thyroid-stimulating immunoglobulin; TBI, thyroid-blocking immunoglobulin. 
Table 1 Comparison of clinical and biochemical characteristics between mild (Group A) and severe (Group B) Graves' orbitopathy

\begin{tabular}{|c|c|c|c|}
\hline Total $(\mathrm{n}=112)$ & $\begin{array}{c}\text { Group } 1 \\
(\mathrm{n}=73,65.2 \%)\end{array}$ & $\begin{array}{c}\text { Group } 2 \\
(\mathrm{n}=39,34.8 \%)\end{array}$ & P-value \\
\hline Age, mean \pm SD & $41.05 \pm 13.04$ & $43.26 \pm 13.37$ & $0.401^{\mathrm{a}}$ \\
\hline Sex ratio, male/female $(\%)$ & $10 / 63(13.7 / 86.3 \%)$ & $9 / 30(23.1 / 76.9 \%)$ & $0.208^{\mathrm{b}}$ \\
\hline Smoker number (\% of group) & $15(20.6 \%)$ & $12(30.8 \%)$ & $0.228^{\mathrm{b}}$ \\
\hline Duration of ocular symptoms at initial diagnosis, median (range) & $3(0.25-6)$ & $3(0.25-6)$ & $0.397^{\mathrm{c}}$ \\
\hline Duration of GD at initial GO diagnosis, median (IQR) & $8(0.25-120)$ & $10(0.5-120)$ & $0.308^{c}$ \\
\hline Duration of ATD treatment, median (range) & $6(0-36)$ & $10(0-60)$ & $0.240^{c}$ \\
\hline Follow up, median (range) & $16.5(12-60)$ & $16(12-45)$ & $0.769^{c}$ \\
\hline Family history, positive ( $\%$ of group) & $11(15.1 \%)$ & $10(25.6 \%)$ & $0.207^{\mathrm{b}}$ \\
\hline Type of GD treatment (no/M/R/S) & $20 / 51 / 2 / 0$ & $6 / 28 / 4 / 1$ & $0.105^{\mathrm{b}}$ \\
\hline $\mathrm{fT} 4(\mathrm{ng} / \mathrm{ml})$, mean $\pm \mathrm{SD}$ & $1.73 \pm 1.40$ & $1.50 \pm 1.12$ & $0.404^{\mathrm{a}}$ \\
\hline Steroid treatment during the follow-up period ( $\%$ of group) & $22(30.1 \%)$ & $27(69.2 \%)$ & $<0.001^{\mathrm{b}}$ \\
\hline Initial CAS, median (IQR) & $2(1)$ & $3(2)$ & $<0.001^{\mathrm{b}}$ \\
\hline Patient no. of increased thyroid size (\% of group) & $25 / 48(52.1 \%)$ & $12 / 23(52.2 \%)$ & $0.994^{\mathrm{b}}$ \\
\hline \multicolumn{4}{|l|}{ Initial TRAb levels } \\
\hline Third-generation TBII, IU/1, mean \pm SD & $7.07 \pm 7.40$ & $21.19 \pm 13.77$ & $<0.001^{\mathrm{a}}$ \\
\hline TSI bioassay, SRR $\%$, mean \pm SD & $320.22 \pm 178.31$ & $597.19 \pm 176.77$ & $<0.001^{\mathrm{a}}$ \\
\hline
\end{tabular}

Abbreviations: CAS, clinical activity score; GD, Graves' disease; GO, Graves' orbitopathy; IQR, interquartile range; M, anti-thyroid drug; R, radioiodine treatment; S, thyroidectomy; SRR, specimen to reference ratio; TBII, thyrotropin-binding inhibitory immunoglobulin; TSI, thyroid-stimulating immunoglobulin.

Bold font is used to denote significant $P$-values. $P$-values $<0.05$ are considered to be statistically significant.

${ }^{\text {a }}$ Based on independent $t$-test.

${ }^{\mathrm{b}}$ Based on Pearson $\chi^{2}$ test.

${ }^{\mathrm{c}}$ Based on Wilcoxon rank-sum test.

between the severe and not severe groups, which was determined by the Youden index. ${ }^{22}$ Statistical analysis was conducted using SAS software (SAS version 9.1; SAS Institute Inc., Cary, NC, USA) and PASS (version 11, NCSS, LLC.; Kaysville, UT, USA), and $P$-values $<0.05$ were considered statistically significant.

\section{Results}

\section{Clinical description}

Of a total of 112 patients, 73 patients $(65.2 \%)$ were determined as having mild GO (Group 1) and 39 patients $(34.8 \%)$ were classified as having severe GO symptoms (Group 2) after 1 year beyond the initial visit. As shown in Table 1, the mean age, the median duration of ocular symptoms at the initial GO diagnosis, type of GD treatment prescribed, and initial mean fT4 level were not different between Groups 1 and 2. The male and smoker proportions were higher in Group 2 than in Group 1, albeit not significantly higher. At initial GO diagnosis, the median prior duration of GD was 8 and 10 months in patients with mild and severe courses, respectively $(P=0.308)$. Of the 73 patients with mild GO, 66 patients (90.4\%) had been treated for GD, using ATDs in 64 patients $(87.7 \%)$ and radioiodine in 2 patients $(2.7 \%)$. The median duration of ATD treatment in mild GO at the initial GO diagnosis was 6 months. Among the 39 patients with severe GO, 30 patients $(76.9 \%)$ had been treated with ATDs, 4 patients (10.3\%) underwent radioiodine treatment, and 1 patient (2.6\%) underwent thyroidectomy. The median duration of ATD treatment in severe GO at the first visit was 10 months. Steroid treatment between the two groups was significantly different $(P=0.003)$, with a much greater proportion of subjects with severe disease (27/39 patients, $69.2 \%$ ) receiving steroids than the subject group with a mild course (22/73 patients, $30.1 \%)$. The median values of initial CAS scores between the two groups was different as well $(P<0.001)$.

\section{Initial TRAb levels (TRAb measured at initial GO diagnosis) in patients with mild and severe GO}

The mean third-generation TBII level was significantly higher in the severe disease Group 2 vs Group 1 (21.19 and $7.07 \mathrm{IU} / 1$, respectively, $P<0.001)$. Likewise, the mean Mc4-CHO TSI level, evaluated by the more specific bioassay, was significantly elevated in Group 2 vs Group 1 (597.19 and 320.22\%, respectively, $P<0.001$, Table 1).The distribution of initial TRAb levels according to mild and severe group was shown in Figures $2 a$ and $b$.

\section{Comparison of the performance levels of the third-generation TBII assay and Mc4-TSI bioassays in predicting the $\mathrm{GO}$ course}

The cutoff values for the prediction of severe course of the third-generation TBII and Mc4-TSI assays were 

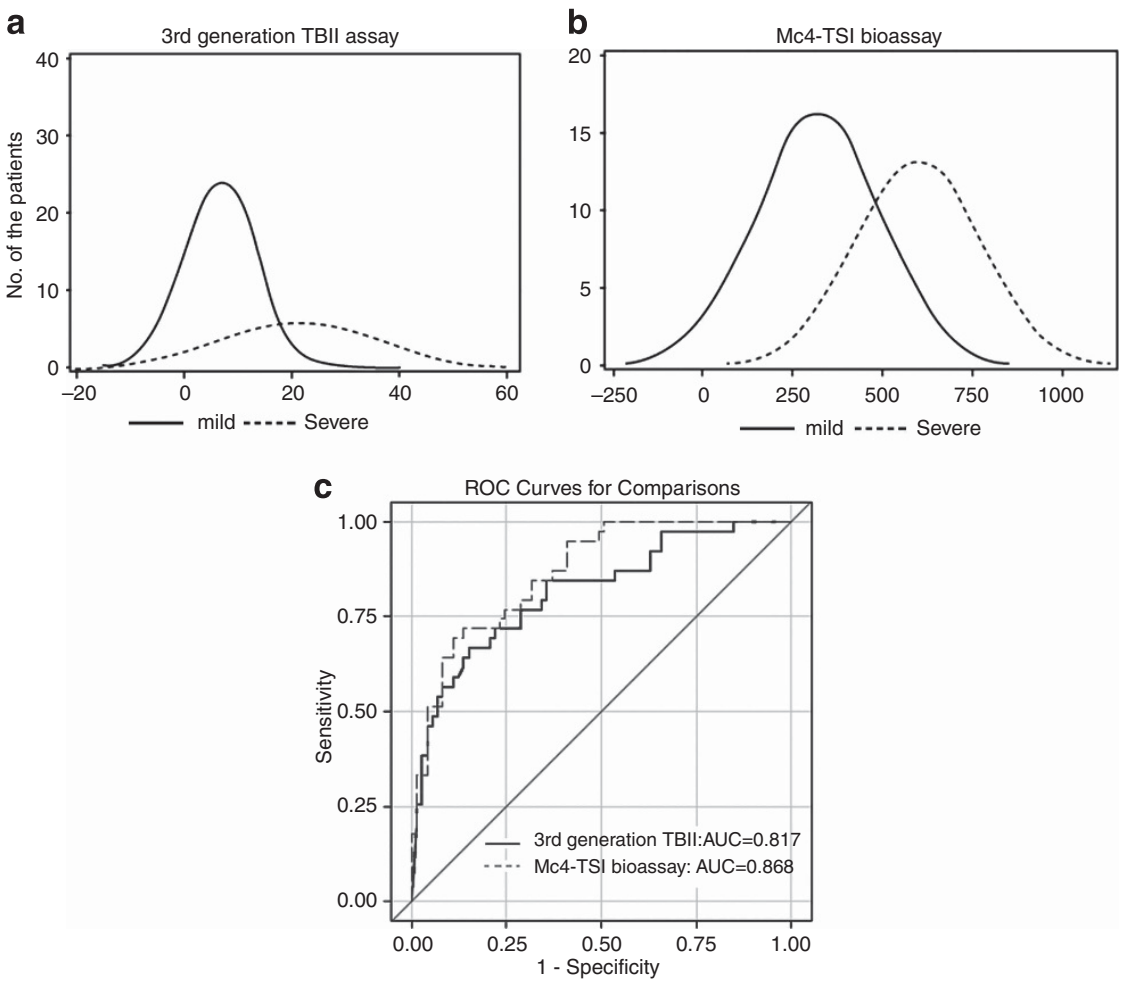

Figure 2 Distribution of TSH receptor autoantibody levels according to mild and severe Graves' orbitopathy, and comparison of the receiver operating characteristic analysis between two different TSH-receptor antibody assays. (a) Distribution of third-generation TBII assay. (b) Distribution of Mc4-TSI bioassay. (c) Comparing areas under the curve for each TRAb assay yielded that the predictive power of the third-generation TBII assay ( $\mathrm{AUC}=0.817,95 \% \mathrm{CI}=0.732-0.902$ ) was equivalent to that of the Mc4-TSI bioassay (AUC $=0.868,95 \% \mathrm{CI}=0.803-0.934)$ in predicting severe patient disease outcome using the Delong method $(P=0.203)$.

Table 2 Cutoff value for the prediction of severe Graves' orbitopathy course of the third-generation TBII and Mc4-TSI bioassay using Youden method

\begin{tabular}{lcc}
\hline & $\begin{array}{c}\text { TBII assay } \\
\geq 10.67 \mathrm{IL} / \mathrm{l}\end{array}$ & $\begin{array}{c}\text { TSI bioassay } \\
\geq 555.10 \%\end{array}$ \\
\cline { 2 - 3 } & $\%(95 \% \mathrm{CI})$ & $\%(95 \% \mathrm{CI})$ \\
\hline Sensitivity & $66.7(51.9-81.5)$ & $69.2(54.7-83.7)$ \\
Specificity & $84.9(76.7-93.1)$ & $89.0(81.9-96.2)$ \\
AUC & $0.817(0.732-0.902)$ & $0.868(0.803-0.934)$ \\
\hline
\end{tabular}

Abbreviations: AUC, area under the curve; CI, confidence interval; TBII, thyrotropin-binding inhibitory immunoglobulin; TSI, thyroid-stimulating immunoglobulin.

10.67 IU / 1 and 555.10 (SRR\%) with respective specificities of 84.9 and $89.0 \%$ and sensitivities of 66.7 and $69.2 \%$

(Table 2). The predictive power of the third-generation TBII assay (AUC $=0.817,95 \%$ confidence interval $(\mathrm{CI})=0.732-0.902)$ was equivalent to that of the Mc4-TSI bioassay $(\mathrm{AUC}=0.868,95 \% \mathrm{CI}=0.803-0.934)$ in predicting severe patient disease outcome $(P=0.203)$ (Table 2 and Figure 2c).

\section{Multiple logistic regression analysis including factors that are associated with the course of GO}

Patients with higher initial TRAb levels had a greater risk of severe disease outcomes. As shown in Table 3, this relationship was independent from age, steroid use, type of GD treatment, fT4, and thyroid size for both the thirdgeneration TBII $(P=0.001)$ and for the Mc4-TSI $(P<0.001)$ assays.

\section{Discussion}

We investigated whether the levels of TRAb measured with third-generation TBII assay and Mc4-TSI bioassay were predictive of clinical courses of GO. Of note, TRAb levels were measured at the time of GO diagnosis, whereas the clinical courses of GO were estimated at least 12 months after the initial diagnosis. We selected newly diagnosed, untreated patients whose duration of ocular symptoms was not longer than 6 months before TRAb measurement. This enabled us to assess the clinical relevance of TRAb at the early stage of GO in terms using TRAb levels to develop an accurate prognosis of the 
Table 3 Multiple logistic regression analysis including factors that are associated with the course of Graves' orbitopathy

\begin{tabular}{|c|c|c|c|c|}
\hline & \multicolumn{2}{|c|}{ Model I (TBII assay) } & \multicolumn{2}{|c|}{ Model II (TSI bioassay) } \\
\hline & OR $(95 \% C I)$ & P-value & OR $(95 \% C I)$ & P-value \\
\hline Age & $1.03(0.98-1.08)$ & 0.334 & $1.05(0.99-1.12)$ & 0.088 \\
\hline Smoker & $3.14(0.42-23.32)$ & 0.263 & $4.80(0.56-40.92)$ & 0.151 \\
\hline \multicolumn{5}{|c|}{ Type of GD treatment } \\
\hline No & 1 & & 1 & \\
\hline M & $1.64(0.20-13.55)$ & 0.646 & $1.33(0.13-13.55)$ & 0.809 \\
\hline $\mathrm{R} / \mathrm{S}$ & $19.66(0.39-988.73)$ & 0.136 & $5.52(0.07-461.31)$ & 0.450 \\
\hline fT4 & $0.69(0.39-1.20)$ & 0.191 & $0.83(0.48-1.43)$ & 0.503 \\
\hline Steroid therapy & $3.18(0.78-12.94)$ & 0.106 & $4.50(0.85-23.76)$ & 0.077 \\
\hline Thyroid size & $1.31(0.30-5.73)$ & 0.721 & $0.73(0.13-4.19)$ & 0.722 \\
\hline TBII assay & $1.14(1.05-1.22)$ & 0.001 & & \\
\hline TSI bioassay & & & $1.01(1.00-1.02)$ & $<0.001$ \\
\hline
\end{tabular}

Abbreviations: CAS, clinical activity score; CI, confidence interval; GD, Graves' disease; $\mathrm{M}$, anti-thyroid drug; OR, odds ratio; R, radioiodine treatment; $\mathrm{S}$, thyroidectomy; TBII, thyrotropin-binding inhibitory immunoglobulin; TSI, thyroid-stimulating immunoglobulin.

TBII and TSI assays were entered into the models I and II, respectively.

Bold font is used to denote significant $P$-values. $P$-values $<0.05$ are considered to be statistically significant.

disease outcome. Interestingly, we found that TRAb levels were significantly higher in patients with a severe course compared with patients with a mild course of GO. Importantly, patients with higher initial levels of TRAb had a significantly higher risk of severe outcomes upon multiple logistic regression analysis. As GO pathogenesis remains incompletely understood and because there are few clues that are currently available to assist clinicians in estimating the course of $\mathrm{GO}, 23-27$ identifying novel, objectively measurable risk factors that could predict severe GO outcomes are of important scientific and clinical benefit. In patients with high levels of TRAb, it would be reasonable to follow up with these patients and monitor the activity and severity more closely. Also regarding the treatment, although the TRAb level alone cannot be an indication of a certain GO treatment, more aggressive anti-inflammatory treatment other than steroid, including radiotherapy ${ }^{28}$ or rituximab ${ }^{29}$ treatment, might be helpful to prevent progression of the disease.

Reporting a positive correlation between changes in TSI and CAS over time, Dragan et al ${ }^{14}$ suggested that serial evaluation of TSI levels would help in deciding which GO patients should get aggressive treatment to prevent a severe inflammatory phase from occurring. Eckstein et $a l^{5}$ reported that persistently high secondgeneration TBII levels were associated with a severe course of GO. Accordingly, serial monitoring of TRAb levels will likely prove to be helpful in the assessment and management of GO. Although we agree that serial measurements of TRAb might provide valuable information, our results suggested that a single TRAb measurement, taken immediately after initial GO diagnosis, would also provide clinicians with a clear impression of whether a patient will present with a mild or severe course in the future.

However, such an association between high TRAb levels and a severe course of GO was not found by Eckstein $e t a l^{5}$ for patients at an early disease stage (within 4 months of first GO symptoms). Based on the results by Laurberg et $a l^{30}$ demonstrating that the largest decrease in TRAb levels occurred within the first 6 months of ATD treatment, we assumed that the discordance between our results and Eckstein et al's might be owing to the difference of the prior GD duration in study subjects. In Eckstein et al's report, ${ }^{5}$ the median GD duration at GO onset in the group with a mild course was 0 months, and 2.4 months in the group with a severe course, indicating that almost all patients required immediate GD treatment initiation. In our study, however, the median duration of thyroid disease was 8 months in the group with mild GO and 10 months in the group with severe GO. In our study, 101 patients $(90.2 \%)$ of the 112 total subjects had already received definitive hyperthyroidism treatment at the time of their initial visit to the ophthalmic clinic. Therefore, highly maintained TRAb levels even after ATD treatment might be associated with GO development and/or progression towards severe GO. This idea is supported by the idea that TRAb consistently mediates the autoimmune process in the orbit. ${ }^{5}$ If TRAb levels were measured before initiating ATD treatment, it might help to elucidate the impact of serial change of TRAb during ATD treatment on GO clinical course.

There are several reports that TSI is more closely associated and reflective of severity of ophthalmopathy than the second-generation TBII assay. ${ }^{17,18}$ A previous report conducted in Singapore ${ }^{31}$ showed that TSI was 
significantly higher in newly diagnosed GD patients with ophthalmic-dominant symptomology, whereas thyroiddominant patients had higher TBII levels. In the present study, ROC plot analysis compared the power of the third-generation TBII and Mc4-TSI bioassays to predict GO progression. Interestingly, the AUC of the thirdgeneration TBII assay was 0.817 and that of the Mc4-TSI assay was 0.868 , which means that both of these TRAb assays have equivalently high power in predicting severe GO disease course. Smith et al ${ }^{12}$ showed the higher sensitivity of the M22-based ELISA than the TSH-based ELISA in diagnosing GD. The human monoclonal TSAb (M22) ${ }^{9,10}$ allows a third-generation TBII assay system where the TRAb inhibits binding of a labeled TSAb rather than labeled TSH to the TSH receptor. Considering that different regions of the TSH receptor are involved in binding TRAb with TSH agonist and antagonist properties, ${ }^{32}$ it was not surprising that the performance level of the third-generation TBII assay was comparable to that of the TSI bioassay.

During the GO active phase, patients should receive immunosuppressive treatment, such as corticosteroids or radiotherapy, to prevent progression to more severe disease and to shorten the active phase. ${ }^{33,34}$ As steroid use could potentially affect the disease course, we considered it necessary to standardize steroid administration in order to accurately evaluate it as a GO risk factor. In our study, no GO patients had been previously treated with steroids at the time of their initial visit. By standardizing our steroid administration protocol, we were able to definitively demonstrate that the association of TRAb with GO course severity is independent of steroid use. Although it is well documented that smoking adversely affects the course of GO, ${ }^{25-27}$ our results did not elucidate this relationship. This might be because there were only 27 smokers and only 39 patients with severe GO in our study. Ethnic differences affect the prevalence and severity of $\mathrm{GO}^{35}$ thus the prevalence of severe course of GO in our study $(34.8 \%)$ was relatively lower than that in Eckstein et al's $(53 \%) .{ }^{5}$ Although the difference did not reach statistical significance owing to small number of smokers in our study, smoking was more common in patients with a severe vs a mild GO course.

In conclusion, both types of TRAb assays, the third-generation TBII assay and the Mc4-TSI assay, were clinically relevant as predictors of a severe course of GO. The predictive power of the third-generation TBII assay was as strong as that of Mc4-TSI bioassay. This is the first report to compare the predictive power of two different laboratory tests for TRAb on GO course severity. We recommend measuring TRAb at the first establishment of a GO diagnosis, in order to better organize future disease management strategies for these patients. Additional studies defining the clinical importance of TRAb in GO are necessary. These include assessing the relationship between TRAb levels and patient responses to medical or surgical GO treatments, and evaluating the utility of TRAb measurement in predicting GO symptom recurrence after rehabilitative surgery.

\section{Summary}

What was known before

- The clinical course of Graves' orbitopathy (GO) is heterogeneous.

- As there are few clues currently available to assist clinicians in estimating GO prognosis, it is very difficult to predict which patients will suffer from such severe sequelae.

What this study adds

- The present study investigated whether the levels of TSH-receptor antibody (TRAb) measured with thirdgeneration TBII assay and Mc4-TSI bioassay were predictive of clinical courses of GO.

- Patients with higher initial TRAb levels had a greater risk of severe disease outcomes. Thus, measurement of TRAb in early GO periods would provide important prognostic information on future GO course.

- The predictive power of the third-generation TBII assay and Mc4-TSI bioassay are similarly strong.

\section{Conflict of interest}

The authors declare no conflict of interest.

\section{Acknowledgements}

We gratefully acknowledge the statistical support of Hye Sun Lee, Biostatistician, Department of Research Affairs, Yonsei University College of Medicine. This research was supported by Basic Science Research Program through the National Research Foundation of Korea (NRF) funded by the Ministry of Education, Science and Technology (7-2011-0250). The sponsor or funding organization had no role in the design or conduct of this research.

\section{References}

1 Bahn RS. Graves' ophthalmopathy. N Engl J Med 2010; 362(8): 726-738.

2 Bahn RS. Clinical review 157: pathophysiology of Graves' ophthalmopathy: the cycle of disease. J Clin Endocrinol Metab 2003; 88(5): 1939-1946.

3 Gorman CA, Bahn RS. Pathogenesis of Graves' ophthalmopathy. Dev Ophthalmol 1989; 20: 1-7.

4 Khoo TK, Bahn RS. Pathogenesis of Graves' ophthalmopathy: the role of autoantibodies. Thyroid 2007; 17(10): 1013-1018.

5 Eckstein AK, Plicht M, Lax H, Neuhauser M, Mann K, Lederbogen $\mathrm{S}$ et al. Thyrotropin receptor autoantibodies are 
independent risk factors for Graves' ophthalmopathy and help to predict severity and outcome of the disease. J Clin Endocrinol Metab 2006; 91(9): 3464-3470.

6 Massart C, Sapin R, Gibassier J, Agin A, d'Herbomez M. Intermethod variability in TSH-receptor antibody measurement: implication for the diagnosis of Graves disease and for the follow-up of Graves ophthalmopathy. Clin Chem 2009; 55(1): 183-186.

7 Vos XG, Smit N, Endert E, Tijssen JG, Wiersinga WM. Frequency and characteristics of TBII-seronegative patients in a population with untreated Graves' hyperthyroidism: a prospective study. Clin Endocrinol (Oxf) 2008; 69(2): 311-317.

8 Kamijo K, Ishikawa K, Tanaka M. Clinical evaluation of 3rd generation assay for thyrotropin receptor antibodies: the M22-biotin-based ELISA initiated by Smith. Endocr J 2005; 52(5): 525-529.

9 Sanders J, Evans M, Premawardhana LD, Depraetere H, Jeffreys J, Richards T et al. Human monoclonal thyroid stimulating autoantibody. Lancet 2003; 362(9378): 126-128.

10 Sanders J, Jeffreys J, Depraetere H, Evans M, Richards T, Kiddie A et al. Characteristics of a human monoclonal autoantibody to the thyrotropin receptor: sequence structure and function. Thyroid 2004; 14(8): 560-570.

11 Pedersen IB, Handberg A, Knudsen N, Heickendorff L, Laurberg P. Assays for thyroid-stimulating hormone receptor antibodies employing different ligands and ligand partners may have similar sensitivity and specificity but are not interchangeable. Thyroid 2010; 20(2): 127-133.

12 Smith BR, Bolton J, Young S, Collyer A, Weeden A, Bradbury J et al. A new assay for thyrotropin receptor autoantibodies. Thyroid 2004; 14(10): 830-835.

13 Gerding MN, van der Meer JW, Broenink M, Bakker O, Wiersinga WM, Prummel MF. Association of thyrotrophin receptor antibodies with the clinical features of Graves' ophthalmopathy. Clin Endocrinol (Oxf) 2000; 52(3): 267-271.

14 Dragan LR, Seiff SR, Lee DC. Longitudinal correlation of thyroid-stimulating immunoglobulin with clinical activity of disease in thyroid-associated orbitopathy. Ophthal Plast Reconstr Surg 2006; 22(1): 13-19.

15 Lytton SD, Kahaly GJ. Bioassays for TSH-receptor autoantibodies: an update. Autoimmun Rev 2010; 10(2): 116-122.

16 Kamijo K, Murayama H, Uzu T, Togashi K, Kahaly GJ. A novel bioreporter assay for thyrotropin receptor antibodies using a chimeric thyrotropin receptor (mc4) is more useful in differentiation of Graves' disease from painless thyroiditis than conventional thyrotropin-stimulating antibody assay using porcine thyroid cells. Thyroid 2010; 20(8): 851-856.

17 Lytton SD, Ponto KA, Kanitz M, Matheis N, Kohn LD, Kahaly GJ. A novel thyroid stimulating immunoglobulin bioassay is a functional indicator of activity and severity of Graves' orbitopathy. J Clin Endocrinol Metab 2010; 95(5): 2123-2131.

18 Ponto KA, Kanitz M, Olivo PD, Pitz S, Pfeiffer N, Kahaly GJ. Clinical relevance of thyroid-stimulating immunoglobulins in graves' ophthalmopathy. Ophthalmology 2011; 118(11): 2279-2285.

19 Bartalena L, Baldeschi L, Dickinson A, Eckstein A, Kendall-Taylor P, Marcocci C et al. Consensus statement of the European Group on Graves' orbitopathy (EUGOGO) on management of GO. Eur J Endocrinol 2008; 158(3): 273-285.

20 Mourits MP, Prummel MF, Wiersinga WM, Koornneef L. Clinical activity score as a guide in the management of patients with Graves' ophthalmopathy. Clin Endocrinol (Oxf) 1997; 47(1): 9-14.

21 Eckstein AK, Lax H, Losch C, Glowacka D, Plicht M, Mann K et al. Patients with severe Graves' ophthalmopathy have a higher risk of relapsing hyperthyroidism and are unlikely to remain in remission. Clin Endocrinol (Oxf) 2007; 67(4): 607-612.

22 Schisterman EF, Perkins NJ, Liu A, Bondell H. Optimal cutpoint and its corresponding Youden index to discriminate individuals using pooled blood samples. Epidemiology 2005; 16(1): 73-81.

23 Bartalena L, Marcocci C, Bogazzi F, Manetti L, Tanda ML, Dell'Unto E et al. Relation between therapy for hyperthyroidism and the course of Graves' ophthalmopathy. N Engl J Med 1998; 338(2): 73-78.

24 Tallstedt L, Lundell G, Torring O, Wallin G, Ljunggren JG, Blomgren $\mathrm{H}$ et al. Occurrence of ophthalmopathy after treatment for Graves' hyperthyroidism. The Thyroid Study Group. N Engl J Med 1992; 326(26): 1733-1738.

25 Bartalena L, Marcocci C, Tanda ML, Manetti L, Dell'Unto E, Bartolomei MP et al. Cigarette smoking and treatment outcomes in Graves ophthalmopathy. Ann Intern Med 1998; 129(8): 632-635.

26 Eckstein A, Quadbeck B, Mueller G, Rettenmeier AW, Hoermann R, Mann K et al. Impact of smoking on the response to treatment of thyroid associated ophthalmopathy. Br J Ophthalmol 2003; 87(6): 773-776.

27 Regensburg NI, Wiersinga WM, Berendschot TT, Saeed P, Mourits MP. Effect of smoking on orbital fat and muscle volume in Graves' orbitopathy. Thyroid 2011; 21(2): 177-181.

28 Tanda ML, Bartalena L. Efficacy and safety of orbital radiotherapy for Graves' orbitopathy. J Clin Endocrinol Metab 2012; 97(11): 3857-3865.

29 Hegedüs L, Smith TJ, Douglas RS, Nielsen CH. Targeted biological therapies for Graves' disease and thyroidassociated ophthalmopathy. Focus on B-cell depletion with Rituximab.. Clin Endocrinol (Oxf) 2011; 74(1): 1-8.

30 Laurberg P, Wallin G, Tallstedt L, Abraham-Nordling M, Lundell G, Torring O. TSH-receptor autoimmunity in Graves' disease after therapy with anti-thyroid drugs, surgery, or radioiodine: a 5-year prospective randomized study. Eur J Endocrinol 2008; 158(1): 69-75.

31 Goh SY, Ho SC, Seah LL, Fong KS, Khoo DH. Thyroid autoantibody profiles in ophthalmic dominant and thyroid dominant Graves' disease differ and suggest ophthalmopathy is a multiantigenic disease. Clin Endocrinol (Oxf) 2004; 60(5): 600-607.

32 Chazenbalk GD, Pichurin P, Chen CR, Latrofa F, Johnstone $\mathrm{AP}$, McLachlan SM et al. Thyroid-stimulating autoantibodies in Graves disease preferentially recognize the free A subunit, not the thyrotropin holoreceptor. J Clin Invest 2002; 110(2): 209-217.

33 De Bellis A, Bizzarro A, Conte M, Coronella C, Solimeno S, Perrino $\mathrm{S}$ et al. Relationship between longitudinal behaviour of some markers of eye autoimmunity and changes in ocular findings in patients with Graves' ophthalmopathy receiving corticosteroid therapy. Clin Endocrinol (Oxf) 2003; 59(3): 388-395.

34 Kahaly GJ, Pitz S, Hommel G, Dittmar M. Randomized, single blind trial of intravenous versus oral steroid monotherapy in Graves' orbitopathy. J Clin Endocrinol Metab 2005; 90(9): 5234-5240.

35 Tellez M, Cooper J, Edmonds C. Graves' ophthalmopathy in relation to cigarette smoking and ethnic origin. Clin Endocrinol (Oxf) 1992; 36(3): 291-294. 